\title{
Les déterminants
}

de l'adoption anticipée des normes comptables internationales: choix financier ou opportunisme? The determinants of the early adoption of international accounting standards: financial decision or opportunism?

Houda Afres* et Antonello Gallimaci**

\section{Résumé}

Le règlement CE 1606/2002 oblige toutes les sociétés cotées de l'union européenne à appliquer le référentiel comptable international à partir du premier janvier 2005. Le but de cette étude est de comprendre le choix de l'adoption anticipée des normes internationales (IAS/IFRS). À l'aide d'un modèle logis-

\section{Abstract}

As of January 2005, rule CE 1606/2002 requires all traded companies of the European Union to use international accounting standards (IAS/IFRS). The motivation of this study is to further understand the choice of early adoption of the IAS/IFRS. Using a logistic model, the research identifies the determinants of early

* Houda Affes est une étudiante diplômée de l'Université du Québec à Montréal.

** Antonello Callimaci est professeur titulaire à l'Université du Québec à Montréal. 
Houda AfFes et Antonello CALlimaCi CHOIX FINANCIER OU OPPORTUNISME?

tique, elle identifie les déterminants de l'adoption anticipée des IAS/IFRS pour un échantillon de 106 entreprises allemandes et autrichiennes. Elle examine également si l'adoption des IAS/IFRS sert d'opportunité pour maquiller la situation financière. Les résultats révèlent que la probabilité d'adoption anticipée des IAS/IFRS augmente avec la taille, l'intensité capitalistique, la dispersion de l'actionnariat et la croissance anormale de la taille et de la rentabilité de la firme. Des analyses supplémentaires permettent de déduire que l'adoption anticipée est motivée par la recherche de financement plutôt que par la manipulation de la situation financière.

MOTS CLÉS. - IAS/IFRS - ADOPTION ANTICIPÉE DÉTERMINANTS D'ADOPTION - THÉORIE DES SIGNAUX, OPPORTUNISME COMPTABLE adoption on a sample of 106 German and Austrian firms. It also examines if the IAS/IFRS adoption is an opportunity to manipulate accounting numbers. The results indicate that size, capital intensiveness, ownership dispersion and the abnormal growth of total assets and $R O E$ increase the likelihood of early adoption. Further analyses allow to infer that the motivation is linked to financing rather than financial statement manipulation.

Correspondance: Antonello Callimaci, Université du Québec à Montréal,

Département des sciences comptables,

C.P. 8888, Succ. Centre-ville, Montréal (Qc), H3C 3P8, Canada

Tel. : 514-987-3000 poste 8938

\section{Introduction}

À l'image des phénomènes de mondialisation et de globalisation des marchés de capitaux de ces dernières années, le monde de la comptabilité s'affere activement à l'harmonisation comptable internationale espérant que cette dernière aboutira en la création de normes comptables susceptibles d'améliorer la comparabilité des états financiers. Le 19 juillet 2002, le parlement et le conseil européens signent le règlement 1606/2002/CE obligeant les entreprises cotées à l'une des bourses de l'Union Européenne (UE) d'appliquer les normes IAS/IFRS pour la préparation des états financiers consolidés à partir du premier janvier 2005. Cette mesure vise, essentiellement, le bon fonctionnement des marchés de capitaux de l'UE et à favoriser l'accès des entreprises à ces derniers. Avant cette échéance, plusieurs entreprises européennes ont choisi d'appliquer les normes internationales pour l'élaboration de leurs états financiers.

L'objectif de cette étude est de comprendre pourquoi certaines entreprises optent pour une adoption anticipée des normes IAS/IFRS alors que d'autres préferent attendre la date limite. De plus, elle examine si l'adoption anticipée résulte d'un plus grand besoin d'accès aux ressources financières sur les marchés de capitaux ou du désir de présenter une meilleure situation financière.

Comptabilité - Contrôle - Audit / Tome ??? - Volume ??? - ??? 2007 (p. ??? à ???) 
Houda AfFes et Antonello CALLimaCi

LES DÉTERMINANTS DE L'ADOPTION ANTICIPÉE DES NORMES COMPTABLES INTERNATIONALES: CHOIX FINANCIER OU OPPORTUNISME?

L'étude traite des déterminants de l'adoption anticipée des IAS/IFRS sur un échantillon d'entreprises allemandes et autrichiennes, les deux pays de l'UE ayant les taux les plus élevés d'adoption anticipée des normes internationales. Elle intègre dans un modèle logistique la taille de l'entreprise, l'intensité capitalistique, la dispersion de la propriété du capital, le niveau d'endettement, la rentabilité, l'internationalité, le type d'auditeur et la croissance de la firme à titre de facteurs susceptibles d'influencer l'adoption anticipée des normes internationales. Malgré les similarités apparentes entre l'Allemagne et l'Autriche, le pays de domiciliation est considéré comme un facteur de contrôle des caractéristiques institutionnelles de chacun des deux pays à l'étude.

Les résultats révèlent que la probabilité d'adoption anticipée des IAS/IFRS augmente avec la taille de l'entreprise, son intensité capitalistique et la dispersion de son actionnariat. Elle augmente également avec le taux de croissance anormale du total des actifs et du rendement sur capitaux propres. La relation de la croissance anormale et de l'adoption anticipée peut être endogène. Par conséquent, en utilisant une mesure de croissance excessive, le papier examine si la croissance anormale est la conséquence ou la cause de l'adoption anticipée. Cette étude établit que les entreprises ayant adopté de façon anticipée les IAS/IFRS ne présentent pas une croissance accrue dans l'année d'adoption. Donc, ces entreprises ne semblent pas se servir de l'adoption des normes pour maquiller leur situation financière. Plutôt, elles s'intéressent à accroître leur accès au financement externe.

Cette étude est la première à considérer et à différencier les motivations menant à l'adoption anticipée des normes internationales. L'identification des caractéristiques des entreprises optant pour l'adoption anticipée des IAS est utile pour les organismes de normalisation. En effet, les résultats semblent démontrer que le comportement des entreprises est cohérent avec l'objectif initial de l'harmonisation qui était de favoriser l'accès des entreprises aux marchés de capitaux européens.

Le papier est organisé comme suit: la première partie résume la revue de littérature traitant du sujet, la seconde énonce les hypothèses de recherche, la troisième décrit la méthodologie utilisée, la quatrième présente les résultats obtenus et leurs discussions, enfin vient la conclusion.

\section{Revue de littérature}

Le but de cette recherche est d'identifier les facteurs susceptibles d'expliquer l'adoption anticipée des normes IAS/IFRS. Les études sur ce sujet sont assez rares. Elles peuvent être regroupées en études traitant de l'adoption anticipée de certaines normes américaines et d'autres traitant de l'adoption volontaire des IAS ${ }^{1}$.

Ayres (1986) examine les facteurs d'adoption anticipée du SFAS 52 portant sur la comptabilisation des devises étrangères. Elle compare 103 firmes américaines ayant anticipé de deux ans l'adoption par rapport à 129 autres l'ayant adopté à la date de mise en application obligatoire. Les résultats dévoilent que les premières sont les plus petites, ont un plus faible taux de croissance des bénéfices pendant la période de pré-adoption, ont un plus faible ratio de recouvrement des intérêts (pour les entreprises fortement endettées) et un ratio de dividendes plus élevé. Ces variables confirment l'hypothèse sous jacente de l'étude, que l'application du SFAS 52 aboutit à une augmentation des résultats. Ainsi, cette étude démontre que l'adoption anticipée d'une norme comptable peut servir d'opportunité pour maquiller la situation financière de l'entreprise.

Comptabilité - Contrôle - Audit / Tome ??? - Volume ??? - ??? 2007 (p. ??? à ???) 
Houda AfFes et Antonello Callimaci CHOIX FINANCIER OU OPPORTUNISME?

Trombley (1989) étudie les caractéristiques de 53 firmes américaines de l'industrie des logiciels dont 14 ont opté pour l'adoption anticipée du SFAS 86 portant sur la capitalisation des frais de développement de logiciels. Le choix d'adoption est examiné en fonction de la taille de l'entreprise et de la position de l'auditeur face à l'adoption. Trombley prend également en considération l'effet d'une baisse des bénéfices de l'année précédente, de l'ampleur de l'endettement et de l'existence d'opérations d'initiés. Il en ressort que la position de l'auditeur est le facteur déterminant dans le choix comptable. Pour les firmes dont l'auditeur ne conseille pas l'adoption anticipée de la norme, la taille devient le facteur le plus influent: les plus petites entreprises sont celles qui adoptent la norme de façon anticipée. Les autres variables de contrôle, à l'exception des opérations d'initiés, sont significatives.

Scott (1991) étudie l'adoption anticipée du SFAS 87 portant sur la comptabilisation des régimes de pensions de retraite. Son échantillon est composé de 279 entreprises et son modèle comporte dix variables explicatives: trois variables politiques (la réglementation des tarifs, les poursuites judiciaires et la taille), quatre variables d'agence (recouvrement des intérêts, politique de dividendes, levier financier, plan d'incitation des dirigeants) et trois variables reliées aux résultats (variation des bénéfices, impact de l'adoption du SFAS 87 et l'effet d'une situation d'assainissement de bilan). Le modèle de Scott explique à $81 \%$ la décision d'adoption anticipée de la nouvelle norme et révèle l'importance des facteurs politiques et de l'impact sur les bénéfices. Sami et Welsh (1992) complètent le travail de Scott (1991). Leurs résultats révèlent encore l'importance des facteurs politiques mais soulèvent, en plus, l'importance des contraintes financières dans les décisions d'adoption d'une nouvelle norme.

Les études sur l'adoption volontaire des normes IAS identifient d'autres variables. Al-Basteki (1995) examine les caractéristiques de 26 firmes cotées du Bahreïn qui ont choisi de divulguer leurs informations selon les IAS. Les hypothèses portent sur cinq caractéristiques: l'influence des auditeurs externes, le type d'industrie, la taille de l'entreprise, l'étendue des opérations avec l'étranger et le degré de dépendance du financement externe. Les résultats montrent que la décision d'adoption des IAS est fortement influencée par la taille de l'auditeur externe (il y a adoption lorsque l'auditeur est un Big 5) ${ }^{2}$. Le type d'industrie semble avoir une certaine influence, mais de moindre importance.

Deux études sont menées sur le cas de multinationales suisses: Dumontier et Raffournier (1998) et Murphy (1999). Ils concluent que le niveau d'activité avec l'étranger (ventes à l'extérieur de la Suisse et cotation sur des marchés étrangers) est le facteur qui différencie clairement le groupe des entreprises adoptant les IAS/IFRS de façon volontaire et celles ne le faisant pas. Dumontier et Raffournier (1998) trouvent également que les coûts politiques, mesurés par la taille et les pressions provenant des marchés de capitaux externes jouent un rôle déterminant dans la décision d'adoption des IAS.

Au niveau de l'Allemagne, Leuz et Verrecchia (2000) investiguent les choix comptables des entreprises cotées sur le DAX index pour l'année 1998. Selon eux, l'adoption des IAS ou des US GAAP signifie opter pour une augmentation de la divulgation financière, une diminution de l'asymétrie d'information et, par conséquent, une diminution du coût en capital. Leur analyse Probit montre que la taille, les besoins de financement et la performance financière favorisent l'adoption de normes reconnues internationalement.

El-Gazzar et al. (1999) et Cuijpers et al. (2002) étudient les caractéristiques des entreprises qui adoptent volontairement des normes internationales (IAS ou US GAAP). L'échantillon d'El-Gazzar $e t$ al. (1999) rassemble 87 firmes dans le groupe expérimental et 87 autres dans le groupe de contrôle. Les résultats montrent que les firmes qui utilisent les normes internationales dans la préparation de

Comptabilité - Contrôle - Audit / Tome ??? - Volume ??? - ??? 2007 (p. ??? à ???) 
Houda AfFes et Antonello CALLimaCi

LES DÉTERMINANTS DE L'ADOPTION ANTICIPÉE DES NORMES COMPTABLES INTERNATIONALES : CHOIX FINANCIER OU OPPORTUNISME?

leurs états financiers ont plus de marchés à l'étranger, sont cotées sur un plus grand nombre de marchés boursiers et sont, en grand nombre, domiciliées dans des pays de l'UE. Cuijpers et al. (2002) se limitent à l'étude des firmes non financières cotées de l'UE. Leur échantillon regroupe 80 entreprises qui ont volontairement adopté les IAS, 34 qui ont volontairement adopté les US GAAP et 1679 entreprises qui utilisent encore les normes locales. Les résultats indiquent que les firmes qui ont plus tendance à adopter des normes internationales (IAS ou US GAAP) sont cotées sur plusieurs marchés, ont un plus grand nombre de segments géographiques, présentent une capitalisation boursière assez élevée, et proviennent de pays où la qualité de l'information est relativement faible.

Ces études indiquent de façon quelque peu mitigée l'existence de liens entre les caractéristiques propres des firmes et l'adoption anticipée des normes comptables. L'adoption anticipée est souvent liée à la taille de l'entreprise, à l'effet de l'adoption de la nouvelle réglementation, et à l'endettement. Les facteurs retenus dans les études sur l'adoption volontaire des normes comptables ne font pas l'unanimité non plus, à l'exception de l'étendue des opérations avec l'étranger et la cotation sur les marchés étrangers. Les variables politiques et les variables financières ne sont pas systématiquement significatives.

\section{Hypothèses de recherche}

Cette étude se base sur les trois propositions suivantes: 1) les IAS/IFRS sont d'une complexité supérieure aux normes domestiques; 2) les IAS/IFRS fournissent de l'information plus complète et de qualité supérieure que les normes domestiques ${ }^{3}$; et par conséquent, 3) l'adoption des normes internationales est un choix de divulgation financière entraînant des coûts non négligeables et pouvant offrir certains bénéfices. Ainsi, il est possible de conclure que l'adoption anticipée est une décision qui résulte d'une analyse coûts/bénéfices. Les entreprises adoptent de façon anticipée les normes internationales lorsqu'elles y trouvent un avantage net, sinon elles retardent l'adoption jusqu'au moment d'y être contraintes. Plusieurs facteurs peuvent expliquer l'adoption anticipée sous cette optique.

\subsection{Taille de l'entreprise}

Selon la théorie des coûts de l'information, le coût de la divulgation d'information détaillée est relativement plus faible pour les grandes firmes que pour les petites. Premièrement, les grandes entreprises produisent souvent de telles informations pour les fins de gestion interne ce qui minimise le coût supplémentaire de production d'information (Buzby, 1975). Deuxièmement, selon la théorie des coûts exclusifs, les rapports financiers des petites firmes sont la source principale d'information pour leurs concurrents et toute divulgation supplémentaire les placerait en désavantage concurrentiel (Raffournier, 1995). Troisièmement, les coûts d'adoption de nouvelles normes peuvent être relativement fixes. Ceci implique que, non seulement, les grandes firmes font face à des coûts moindres de divulgation, mais, de plus, elles sont plus aptes à supporter ou amortir ces coûts (Cuijpers et al. 2002; Street et Gray, 2002; Al-Basteki, 1995).

Singhvi et Desai (1971) ajoutent que les plus grandes firmes considèrent davantage la nécessité d'adopter des normes internationalement acceptées. Les grandes firmes sont plus dépendantes des capitaux externes et cherchent à se distinguer sur le marché en donnant de l'information financière

Comptabilité - Contrôle - Audit / Tome ??? - Volume ??? - ??? 2007 (p. ??? à ???) 
Houda AfFes et Antonello CALLimaCi CHOIX FINANCIER OU OPPORTUNISME?

de qualité supérieure. Par exemple, en Allemagne et en Autriche, l'adoption des IAS/IFRS rend plus difficile la gestion des bénéfices vu l'interdiction de la constitution de réserves latentes (contrairement à la réglementation locale); les bénéfices présentés selon les normes internationales sont considérés de qualité supérieure (Bartov et al., 2002). La première hypothèse est donc la suivante:

$H_{1}$ : Plus une entreprise est grande, plus elle est favorable à l'adoption anticipée des IAS/IFRS.

\subsection{Intensité capitalistique}

Les firmes à forte intensité capitalistique doivent investir des sommes habituellement considérables en ayant recours à un grand nombre de parties prenantes. Ces firmes sont alors obligées d'avoir un niveau de divulgation qui satisfait les besoins d'un grand nombre d'utilisateurs de leurs états financiers. Il est également possible que le financement d'importants investissements en capital provienne principalement de gros intermédiaires financiers ayant un accès privilégié à l'information financière de l'entreprise et pour qui la rigueur accrue des normes IAS aurait peu d'importance. Ainsi, dans ce dernier cas, les sociétés préferent éviter les coûts exclusifs d'une divulgation accrue.

À la lumière de la présente discussion, les deux explications prédisent des relations de signes opposés, il n'est pas étonnant que Chow et Wong-Boren (1987), Dumontier et Raffournier (1998) et Hossain et al. (1994) trouvent qu'il n'existe pas de relation significative entre l'intensité capitalistique et la divulgation volontaire d'information. Étant donné que l'intensité capitalistique peut également mesurer les besoins de financement et puisque l'adoption des IAS/IFRS permet l'accès à un plus grand nombre de sources de financement, l'hypothèse suivante parait plus plausible:

$\mathrm{H}_{2}$ : Plus une entreprise a une forte intensité capitalistique, plus elle est favorable à l'adoption anticipée des IAS/IFRS.

\subsection{Dispersion de l'actionnariat}

La littérature fait la distinction entre les firmes ayant un capital-actions dispersé et celles ayant un capital-actions concentré. Dumontier et Raffournier (1995) affirment que la conformité aux IAS/IFRS peut être considérée comme une décision choisie par les managers. Ces derniers visent à réduire les coûts d'agence en diminuant le coût de la recherche d'information par les petits investisseurs ou le coût d'activités de contrôle imposées par les actionnaires. Selon Cuijpers et al. (2002), les firmes, dont la majorité du capital est détenue par les dirigeants, ont moins de motivation à divulguer une information de bonne qualité puisque les actionnaires sont les détenteurs même de l'information. Conformément aux hypothèses de la théorie du coût de l'information et de la théorie de l'agence, l'hypothèse suivante est testée:

$H_{3}$ : Plus la propriété du capital-actions de l'entreprise est dispersée, plus elle est favorable à l'adoption anticipée des IAS/IFRS.

\subsection{L'endettement}

Même si une relation entre l'endettement et l'adoption volontaire des IAS/IFRS peut sembler raisonnable du point de vue théorique, les résultats empiriques ne sont pas toujours concluants. Par exemple, Murphy (1999) et Dumontier et Raffournier (1998) ne trouvent aucune relation significa-

Comptabilité - Contrôle - Audit / Tome ??? - Volume ??? - ??? 2007 (p. ??? à ???) 
Houda AfFes et Antonello CALLimaCi

LES DÉTERMINANTS DE L'ADOPTION ANTICIPÉE DES NORMES COMPTABLES INTERNATIONALES : CHOIX FINANCIER OU OPPORTUNISME?

tive entre les deux. Par contre, Meek et al. (1995) suggèrent que la divulgation volontaire d'information augmente avec le levier financier puisque les firmes les plus endettées ont des coûts d'agence plus élevés et cherchent à les minimiser en dévoilant plus d'informations utiles à leurs créanciers. De plus, les entreprises très endettées peuvent être motivées à adopter des normes de meilleure qualité afin de rassurer les créanciers qu'elles ne contourneront pas les clauses restrictives par des pratiques comptables douteuses. Finalement, un niveau élevé d'endettement peut être un indicateur de la nécessité, pour l'entreprise, de trouver de nouvelles sources de financement; l'adoption des IAS étant un moyen d'accéder à certaines d'entre elles. L'hypothèse posée est la suivante:

$H_{4}$ : Plus une entreprise est endettée, plus elle est favorable à l'adoption anticipée des IAS/IFRS.

\subsection{Le rendement}

Singhvi et Desai (1971) affirment que des rendements croissants motivent les gestionnaires à divulguer davantage d'informations afin de rassurer les investisseurs que l'entreprise est réellement rentable. En éliminant l'utilisation de réserves arbitraires, les normes IAS/IFRS rendent plus difficile la gestion des bénéfices. L'adoption anticipée des normes IAS/IFRS permet de signaler que la performance financière supérieure de la firme est authentique et ne provient pas d'artifice comptable. Les résultats empiriques sont mitigés au sujet de la relation entre le rendement et la divulgation volontaire: Singhvi et Desai (1971) établissent une relation positive, Wallace et Naser (1995) suggèrent une relation négative alors que Dumontier et Raffournier (1998) ne trouvent aucun lien significatif. Conformément aux résultats de Singhvi et Desai (1971), l'hypothèse posée est:

$H_{5}$ : Plus une entreprise est rentable, plus elle est favorable à l'adoption anticipée des IAS/IFRS.

\subsection{L'internationalité}

La publication d'informations conformes aux normes IAS/IFRS épargne aux multinationales le coût de la divulgation multiple, permet de réduire leur coût du capital (Leuz et Verrecchia, 2000) et les aide à obtenir de meilleures conditions de financement (Dumontier et Raffournier, 1998). Plusieurs études constatent que l'internationalité, c'est-à-dire, le fait d'avoir d'importantes opérations à l'étranger, est positivement associé avec l'adoption volontaire des normes internationales (Dumontier et Raffournier, 1998; Murphy, 1999; Cuijpers et al., 2002; El-Gazzar et al., 1999). Uniquement quelques études ne trouvent pas de relation significative (par exemple, Al-Basteki, 1995). Ainsi, l'hypothèse suivante prévoit une relation positive:

$H_{6}$ : Plus une entreprise réalise des opérations avec l'étranger, plus elle est favorable à l'adoption anticipée des IAS/IFRS.

\subsection{Le type d'auditeur}

Singhvi et Desai (1979) suggèrent que plus la firme d'audit est grande et réputée, plus elle encourage ses entreprises clientes à divulguer de l'information. Les grandes firmes d'audit détiennent, peut-être, une plus grande compétence au niveau de la comptabilité internationale étant donné la formation de leurs professionnels. Elles peuvent réaliser des économies d'échelles en appliquant les mêmes normes internationales pour la majorité de leurs clients (Dumontier et Raffournier, 1998; Al-Basteki, 1995;

Comptabilité - Contrôle - Audit / Tome ??? - Volume ??? - ??? 2007 (p. ??? à ???) 
Houda AfFes et Antonello CALLimaCi CHOIX FINANCIER OU OPPORTUNISME?

Murphy, 1999; Mora et Rees, 1998). Il est également possible que les entreprises qui envisagent l'adoption des normes IAS/IFRS changent d'auditeur pour pouvoir bénéficier de l'expertise de ces grands cabinets. Pour une raison ou une autre, la relation prévue est positive et l'hypothèse testée est la suivante:

$H_{7}$ : Une entreprise auditée par un grand cabinet est plus favorable à l'adoption anticipée des IAS/IFRS.

\subsection{Les variables de croissance anormale}

Selon Lang et Lundholm (1993), les caractéristiques reliées à la structure et à la performance de l'entreprise affectent la quantité d'information financière divulguée. Ainsi, la croissance de ces caractéristiques devrait, également, influencer la quantité d'information financière divulguée. En conséquence, selon la deuxième proposition, elle devrait influencer l'adoption anticipée des normes IAS/IFRS. La croissance anormale de la taille, du niveau d'endettement et du rendement sont utilisées afin de capturer les caractéristiques structurelles et de performance. La croissance anormale est celle qui dévie de la croissance du marché et permet ainsi de contrôler pour l'évolution générale du marché (Ball et Brown, 1968). Le lien entre les trois croissances anormales choisies et l'adoption anticipée des IAS/IFRS est expliqué ci-dessous.

Les dirigeants d'entreprises, qui soutiennent une croissance importante, peuvent adopter les IAS/IFRS afin de faciliter l'accès à un plus grand nombre de ressources financières, nécessaires à cette croissance. Cette logique mène à prédire une relation positive entre l'adoption anticipée des IAS/IFRS et la croissance anormale de la taille. Cette même logique s'applique à la croissance anormale de l'endettement, menant aussi à une relation positive avec l'adoption anticipée des IAS/IFRS. De plus, il est possible que les dirigeants soient soucieux de diminuer le niveau d'endettement présenté aux états financiers. Les normes IAS/IFRS sont moins conservatrices que les normes locales de l'Allemagne et de l'Autriche et sont susceptibles de révéler un niveau d'endettement moins élevé. En effet, Mora et Rees (1998) trouvent que l'adoption de la consolidation conformément aux IAS/IFRS diminue le niveau d'endettement des entreprises espagnoles. Une autre logique suggère que les dirigeants de sociétés dont l'endettement croît à un niveau supérieur à celui du marché doivent rassurer leurs créanciers que cet accroissement ne s'accompagne pas d'une détérioration de la situation financière. Ils tenteront d'améliorer la qualité de la divulgation financière pour montrer que l'entreprise est toujours en mesure d'honorer ses engagements (Wallace et Naser, 1995). Toutes ces explications mènent à prédire une relation positive entre l'adoption des normes IAS/IFRS et la croissance anormale de la taille et de l'endettement.

$H_{8}$ : Plus une entreprise exhibe une croissance anormale de sa taille, plus elle est favorable à l'adoption anticipée des IAS/IFRS.

$H_{9}$ : Plus une entreprise exhibe une croissance anormale de son endettement, plus elle est favorable à l'adoption anticipée des IAS/IFRS.

Lang et Lundholm (1993) suggèrent que le niveau de divulgation augmente en fonction de l'asymétrie d'information et que la variation de la performance est un indicateur de cette asymétrie. À partir d'un échantillon de 2272 entreprises-années, ils trouvent que les firmes à rendement très variable divulguent plus d'information afin de diminuer l'asymétrie d'information. Ainsi, les entreprises dont la rentabilité augmente peuvent vouloir présenter plus d'information dans leurs rapports

Comptabilité - Contrôle - Audit / Tome ??? - Volume ??? - ??? 2007 (p. ??? à ???) 
Houda AfFes et Antonello CALLimaCi

LES DÉTERMINANTS DE L'ADOPTION ANTICIPÉE DES NORMES COMPTABLES INTERNATIONALES: CHOIX FINANCIER OU OPPORTUNISME?

annuels afin de diminuer l'asymétrie d'information et d'en augmenter la crédibilité. Étant donné que les états financiers dressés selon les normes IAS/IFRS présentent une information plus complète et de meilleure qualité, cette étude prédit que les entreprises réalisant des taux élevés de croissance anormale de rendement sont plus susceptibles à adopter de façon anticipée les normes IAS/IFRS. Ainsi la dernière hypothèse concernant la croissance anormale est la suivante:

$H_{10}$ : Plus une entreprise exhibe une croissance anormale de son rendement, plus elle est favorable à l'adoption anticipée des IAS/IFRS.

\section{Méthodologie}

\subsection{Mesure des variables et présentation du modèle}

Toutes les variables précitées, taille, intensité capitalistique, dispersion de l'actionnariat, endettement, rendement, internationalité, type de l'auditeur, croissance anormale de la taille, croissance anormale de l'endettement et croissance anormale du rendement, sont intégrées dans le modèle logistique présenté ci-dessous.

$$
\begin{aligned}
& \mathrm{ADOP}_{\text {it }}=\beta_{0}+\beta_{1} \mathrm{TACT}_{\text {it }}+\beta_{2} \mathrm{ICAP}_{\text {it }}+\beta_{3} \mathrm{DISP}_{\mathrm{it}}+\beta_{4} \mathrm{LEV}_{\mathrm{it}}+\beta_{5} \mathrm{ROE}_{\mathrm{it}}+\beta_{6} \mathrm{COTA}_{\mathrm{it}} \\
& +\beta_{7} \text { AUDIT }_{\text {it }}+\beta_{8} \text { PAYS }_{\text {it }}+\beta_{9} \text { CTACT }_{\text {it }}+\beta_{10} \text { CLEV }_{\text {it }}+\beta_{11} \text { CROE }_{\text {it }}+\beta_{\text {it }}
\end{aligned}
$$

La variable dépendante (ADOP) est égale à 1 lorsque l'entreprise a adopté de façon anticipée les normes IAS/IFRS (0 lorsque l'entreprise utilise les normes locales). La taille est mesurée par le log base $10 \mathrm{du}$ total des actifs (TACT) ${ }^{4}$. L'intensité capitalistique (ICAP) est calculée par la proportion des immobilisations corporelles nettes sur le total des actifs. La dispersion de l'actionnariat (DISP) est mesurée par la formule suivante: 1 moins la proportion de titres détenus par un groupe lié d'actionnaires. Le ratio du total des emprunts divisé par le total des actifs représente l'endettement (LEV). Le rendement est mesuré par le rendement des capitaux propres (ROE); il est égal au résultat net divisé par les capitaux propres. L'internationalité (COTA) est une variable dichotomique faisant la distinction entre les entreprises cotées uniquement sur le marché local $(C O T A=0)$ et celles cotées sur plus d'un marché $(\mathrm{COTA}=1)$. La variable audit (AUDIT) est également dichotomique et prend la valeur 1 lorsque l'auditeur de l'entreprise est un grand cabinet d'audit ( 0 pour tout autre cabinet). Les grands cabinets d'audit correspondent au Big 5.

La mesure des variables de croissance anormale est empruntée au modèle de prévision des bénéfices de Ball et Brown (1968). Elle tient compte, non seulement des données antérieures de la firme mais aussi de celles du marché. Le taux de croissance anormale d'une variable est la différence entre la valeur observée et la valeur anticipée, le tout divisé par la valeur anticipée:

$$
\frac{\text { Valeur observée }_{\text {it }}-\text { Valeur anticipée }_{\text {it }}}{\text { Valeur anticipée }_{\text {it }}}
$$

La valeur anticipée au temps $t$ correspond à la valeur observée au temps $t-1$ majorée par la croissance réelle du marché domestique pour la période de $t-1$ à $t$. Ainsi, la valeur anticipée représente la valeur qu'une entreprise aurait réalisée en suivant la tendance des entreprises de son pays. Donc, la

Comptabilité - Contrôle - Audit / Tome ??? - Volume ??? - ??? 2007 (p. ??? à ???) 
Houda AfFes et Antonello CALLimaCi CHOIX FINANCIER OU OPPORTUNISME?

croissance anormale pour chacune des trois variables (taille, endettement et rendement) est calculée de la façon suivante:

$$
\frac{\mathrm{V}_{\mathrm{it}}-\left[\mathrm{V}_{\mathrm{it}-1} \cdot\left(\mathrm{V}_{\mathrm{mt}} / \mathrm{V}_{\mathrm{mt}-1}\right)\right]}{\mathrm{V}_{\mathrm{it}-1} \cdot\left(\mathrm{V}_{\mathrm{mt}} / \mathrm{V}_{\mathrm{mt}-1}\right)}
$$

où $V$ est la mesure désirée de la taille, l'endettement ou du rendement, pour l'entreprise $i$ de notre échantillon au temps $t$, soit l'année d'adoption des IAS/IFRS 5 . Le temps $t-1$ est l'année précédente et $m$ représente la valeur médiane de toutes les entreprises constituant le marché domestique.

Finalement, le pays de domiciliation est intégré en tant que variable factice (PAYS) afin de contrôler l'effet des facteurs externes propres à chacun des deux pays à l'étude (réglementation, culture, système fiscal, niveau de développement économique, etc.) La variable Pays prend la valeur de 1 pour l'Autriche (0 pour l'Allemagne).

\section{2. Échantillon}

L'échantillon est composé exclusivement de sociétés autrichiennes et allemandes. Ce choix permet d'éviter le biais inhérent aux études regroupant des sociétés provenant de pays à différences institutionnelles marquées. En effet, une étude dirigée par Pricewaterhouse Coopers révèle que l'Autriche et l'Allemagne sont les leaders de l'UE en matière d'adoption anticipée des IAS/IFRS. La sélection du groupe expérimental est faite à partir de trois sources: le site officiel de l'IASB, l'ouvrage de David Cairns "International Accounting Standards, Survey 2000 » et la base de données Worldscope. Pour être retenues, les entreprises doivent remplir trois conditions: a) leur utilisation des IAS/IFRS doit être complète ${ }^{6}$ et définitive ${ }^{7}$ tel qu'indiqué dans les rapports annuels; b) leurs données doivent être disponibles sur la base de données Worldscope; c) elles doivent être cotées sur au moins un marché financier européen puisque le règlement 1606/2002/CE ne s'applique qu'aux entreprises faisant appel public à l'épargne.

Worldscope sert de point de départ pour la sélection du groupe de contrôle. Il s'agit de prendre des entreprises allemandes et autrichiennes cotées en bourse, qui ne sont pas des institutions financières et qui utilisent des normes locales tel que mentionné dans leur rapport annuel ${ }^{8}$. Pour chaque entreprise du groupe expérimental, une entreprise de contrôle est attribuée aléatoirement en respectant les trois critères d'appariement suivants: son pays de domiciliation, son activité principale et l'année d'observation. L'échantillon initial est constitué de 126 entreprises (94 allemandes et 32 autrichiennes) et est parfaitement apparié, 63 ont adopté les IAS/IFRS de façon anticipée et 63 utilisent encore les normes locales.

Suite à une première analyse des données, neuf (9) observations considérées aberrantes sont éliminées. Une observation est aberrante si son poids est égal ou supérieur à $10 \%$ de la somme des valeurs absolues de l'échantillon pour une des variables suivantes: la taille, le ROE et les variables de croissance ${ }^{9}$. De plus, suite à une analyse préliminaire du modèle, les observations pour lesquelles les résidus standardisés sont supérieurs à 2 écart-types de la moyenne sont éliminées. Ceci permet d'identifier 11 autres observations aberrantes. Ainsi, l'échantillon passe de 126 à 106 entreprises. L'échantillon final est équilibré mais non plus parfaitement apparié: 53 entreprises qui utilisent les normes IAS/IFRS et 53 qui continuent à utiliser les normes locales ${ }^{10}$. 
Houda AfFes et Antonello CALLimaCi

LES DÉTERMINANTS DE L'ADOPTION ANTICIPÉE DES NORMES COMPTABLES INTERNATIONALES : CHOIX FINANCIER OU OPPORTUNISME?

\section{Analyse des résultats}

\subsection{Corrélations et analyses univariées}

Une analyse des corrélations de Pearson entre les variables indépendantes ne dévoile pas de problèmes majeurs ${ }^{11}$. La seule préoccupation concerne la taille et sa croissance (TACT et CTACT) qui sont significativement corrélées avec plusieurs autres variables explicatives. L'une ou l'autre de ces mesures est corrélée à l'internationalité (COTA), le type d'auditeur (AUDIT) et l'endettement (LEV et CLEV). Pour s'assurer que cela n'affecte pas indument les résultats, l'analyse logistique a été reproduite sans les variables de la taille (TACT et CTACT). Les résultats demeurent sensiblement inchangés $^{12}$. Deux autres corrélations sont significatives: le rendement (ROE) et l'endettement (LEV) sont respectivement corrélés avec leur croissance anormale (CROE et CLEV). Pour en évaluer l'impact sur les résultats, des tests de robustesse ont été exécutés sans les variables de croissance. À nouveau, sensiblement, les mêmes résultats sont obtenus. En conclusion, les corrélations entre les variables indépendantes ne sont pas problématiques.

À titre d'analyse préliminaire, les deux groupes sont comparés à l'aide d'analyses univariées ${ }^{13}$. Des tests d'égalité des moyennes ( $t$-test) sont menés pour les variables cardinales et des tests d'indépendance de Chi carré $\left(\chi^{2}\right)$ pour les variables dichotomiques. Les analyses révèlent une supériorité significative de la taille (TACT), de l'intensité capitalistique (ICAP) et de la croissance anormale de l'endettement (CLEV) des entreprises ayant adopté les normes IAS/IFRS. Les résultats montrent également, de façon marginalement significative, une plus grande dispersion de l'actionnariat (DISP) et une plus grande croissance de l'actif total (CTACT) du groupe expérimental. Le test de $\chi^{2}$ révèle que la proportion des entreprises ayant adopté les normes internationales, qui sont auditées par un grand cabinet, est significativement supérieure à celle des entreprises utilisant les normes locales. Tous ces résultats sont conformes aux attentes.

\subsection{Analyse logistique}

L'analyse logistique présentée au tableau 1 confirme les résultats de celle univariée. Le modèle est globalement significatif et classe correctement 73,6\% des observations. Conformément à l'hypothèse 1 , le coefficient positif et significatif de la taille $\left(\log\right.$ TACT, $\left.\beta_{1}=1,285 ; p=0,004\right)$ indique que les grandes entreprises sont les plus propices à l'adoption anticipée des IAS/IFRS. Ce résultat peut découler du fait que les grandes firmes font face à des coûts moindres de divulgation, conformément à la théorie des coûts exclusifs, et possèdent la capacité financière pour absorber les coûts d'adoption. Une deuxième explication est possible: les grandes entreprises doivent fournir une information financière de qualité supérieure afin de s'assurer l'accès à des sources plus importantes et variées de financement. L'intensité capitalistique (ICAP) est également positivement et significativement liée à l'adoption des IAS/IFRS $\left(\beta_{2}=0,039 ; \mathrm{p}=0,021\right)$ confirmant l'hypothèse 2 selon laquelle les entreprises à forte intensité capitalistique sont les plus favorables à l'adoption des IAS/IFRS. Ce résultat peut s'expliquer de deux façons: soit par les coûts de la divulgation soit par des exigences de financement. Premièrement, les entreprises à forte intensité capitalistique sont, habituellement, financièrement solides et disposent des moyens pour absorber les coûts de la divulgation d'information supplémentaire. Deuxièmement, ces entreprises subissent plus de pressions pour la production d'une infor-

Comptabilité - Contrôle - Audit / Tome ??? - Volume ??? - ??? 2007 (p. ??? à ???) 
Houda AfFes et Antonello Callimaci CHOIX FINANCIER OU OPPORTUNISME?

mation de meilleure qualité puisqu'elles ont plus recours au financement à long-terme. Les résultats concernant la dispersion de l'actionnariat (DISP) permettent de renforcer l'importance du financement comme explication de l'adoption anticipée. Tel que prévu à l'hypothèse 3 , les entreprises dont la propriété du capital est plus dispersée sont les plus propices à l'adoption anticipée des IAS/IFRS $\left(\beta_{3}=0,030 ; p=0,017\right)$. Cela semble confirmer que les entreprises à actionnariat dispersé désirent diminuer leurs coûts d'agence (et donc de financement) en divulguant une information de meilleure qualité. Cela peut également s'expliquer par le fait que la dispersion de l'actionnariat est synonyme de quête de financement externe. Ce résultat renforce l'explication suggérée par les deux premières hypothèses: l'adoption anticipée des normes IAS/IFRS peut être fortement motivée par un besoin de financement.

\section{Tableau 1}

\section{Analyse logistique des déterminants de l'adoption anticipée des normes IAS/IFRS Modèle de base}

\begin{tabular}{|c|c|c|c|}
\hline \multicolumn{4}{|c|}{ 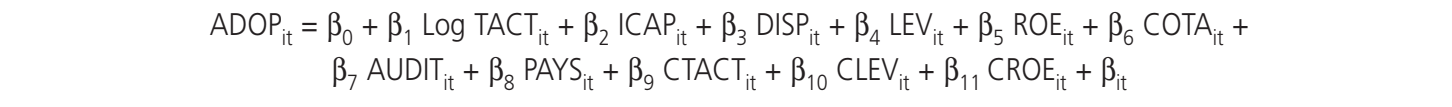 } \\
\hline Variable & Signe prévu & Coefficient $\beta$ & $p$ \\
\hline Log TACT & + & 1,285 & 0,004 \\
\hline ICAP & + & 0,039 & 0,021 \\
\hline DISP & + & 0,030 & 0,017 \\
\hline LEV & + & $-0,006$ & 0,789 \\
\hline ROE & + & $-0,017$ & 0,137 \\
\hline COTA & + & 0,541 & 0,564 \\
\hline AUDIT & + & $-0,961$ & 0,104 \\
\hline PAYS & $+/-$ & $-0,396$ & 0,523 \\
\hline CTACT & + & 0,019 & 0,024 \\
\hline CLEV & + & $-0,001$ & 0,512 \\
\hline CROE & + & 0,054 & 0,063 \\
\hline \multicolumn{2}{|c|}{ Taux de prédiction non biaisé: 73,6 \% } & N: 106 & $R^{2}$ de Nagelkerke: $44,9 \%$ \\
\hline \multicolumn{4}{|c|}{$\begin{array}{l}\text { Pour fins de concision les indices i pour l'entreprise et t pour l'exercice de clôture sont omis. La variable ADOP est égale } \\
\text { à } 1 \text { lorsque l'entreprise a adopté de façon anticipée les normes IAS/IFRS; Log TACT est le logarithme base } 10 \text { du total des } \\
\text { actifs; ICAP est la proportion des immobilisations corporelles sur le total des actifs; DISP est le résultat de } 1 \text { moins la pro- } \\
\text { portion de titres détenus par un groupe lié; LEV est le total des emprunts divisé par le total des actifs; ROE est le résul- } \\
\text { tat net divisé par les capitaux propres; COTA est égale à } 1 \text { lorsque l'entreprise est cotée sur plus d'un marché; AUDIT est } \\
\text { égale à } 1 \text { lorsque l'auditeur est un Big } 5 \text {; PAYS est égale à } 1 \text { pour l'Autriche et } 0 \text { pour l'Allemagne; CTACT, CLEV et CROE } \\
\text { représentent respectivement la croissance anormale des variables TACT, LEV et ROE. La croissance anormale d'une variable } \\
\text { V est mesurée par: }\left[V_{i t}-\left(V_{i t-1} *\left(V_{m t} / V_{m t-1}\right)\right] /\left[V_{\text {it-1 }} *\left(V_{m t} / V_{m t-1}\right)\right] ; V_{m t} \text { étant la valeur médiane de V. }\right.\end{array}$} \\
\hline
\end{tabular}

En ce qui concerne l'endettement (LEV), le rendement (ROE), l'internationalité (COTA) et le type d'auditeur (AUDIT), les coefficients de ces variables ne sont pas significatifs (respectivement, $\mathrm{p}=0,789, \mathrm{p}=0,137, \mathrm{p}=0,564$ et $\mathrm{p}=0,104)$. Le fait que l'endettement et le rendement ne soient pas significatifs, n'est pas unique à cette recherche (voir Dumontier et Raffournier (1998), par exemple). Le résultat que l'internationalité n'affecte pas la propension à l'adoption anticipée des normes internatio- 
Houda AfFes et Antonello CALlimaci

LES DÉTERMINANTS DE L'ADOPTION ANTICIPÉE DES NORMES COMPTABLES INTERNATIONALES : CHOIX FINANCIER OU OPPORTUNISME?

nales, semble étonnant à première vue. Tel que mentionné précédemment, plusieurs auteurs (Dumontier et Raffournier, 1998; Murphy, 1999; Cuijpers et al., 2002; El-Gazzar et al., 1999) ont trouvé une relation positive et significative entre l'internationalité et l'adoption volontaire des normes internationales. La différence entre les résultats de cette recherche et ceux des auteurs précités peut s'expliquer par le fait que les adoptions anticipées et volontaires ne représentent pas la même situation. Une adoption anticipée est une adoption qui a lieu avant qu'elle ne devienne obligatoire alors qu'une adoption volontaire est entièrement facultative. Il semble donc cohérent que les entreprises à vocation plus internationale soient plus favorables à adopter volontairement des normes internationales. Dans le cas d'une éventuelle adoption obligatoire, même les entreprises sans vocation internationale peuvent avoir des motivations importantes à anticiper l'adoption des normes IAS/IFRS. Le résultat obtenu est en accord avec un des objectifs de l'harmonisation des normes comptables qui est de fournir de l'information de qualité comparable par toutes les entreprises, qu'elles soient locales ou internationales. La variable AUDIT indique que les entreprises auditées par des Big 5 ne sont pas plus favorables que les autres à adopter de façon anticipée les normes IAS/IFRS. Il est fort possible que les cabinets internationaux de taille moyenne ${ }^{14}$ et même certains grands cabinets locaux aient suffisamment d'expertise pour concurrencer les Big 5 lorsqu'il s'agit d'assister les entreprises cotées à adopter les normes IAS/IFRS.

Tel que prévu aux hypothèses huit (8) et dix (10), les croissances anormales dans l'année d'adoption de la taille (CTACT) et du rendement (CROE) présentent des coefficients positifs (respectivement $\beta_{9}=0,019$ et $\beta_{11}=0,054$ ) et significatifs (respectivement $\mathrm{p}=0,024$ et $\mathrm{p}=0,063$ ). Ceci suggère que les entreprises ayant une croissance de la taille et/ou du rendement supérieure à celle du marché sont les plus propices à l'adoption anticipée des IAS/IFRS. Ces résultats s'expliquent de deux façons: i) soit la croissance est naturelle et les IAS/IFRS servent de moyen pour accéder aux ressources financières nécessaires pour alimenter cette croissance, ou ii) soit elle est la conséquence comptable de l'adoption visée sciemment par la firme. L'authenticité de ces deux explications est étudiée dans la sous-section ci-dessous.

Tout comme la variable de l'endettement, celle de sa croissance anormale (CLEV) n'est pas significative $(p=0,512)$. L'adoption anticipée des normes IAS/IFRS semble avoir peu d'importance pour les entreprises fortement endettées puisqu'il est possible que leurs créanciers exigent le respect de clauses restrictives basées sur des calculs spécifiques. Finalement, il faut noter que le pays de domiciliation (Allemagne ou Autriche) n'influence pas la décision d'adoption des normes IAS/IFRS ( $\mathrm{p}=0,523$ ).

\subsection{Analyses additionnelles des croissances anormales}

Les résultats soulignent que les entreprises qui adoptent de façon anticipée les IAS/IFRS sont celles qui ont une croissance anormale supérieure de la taille et du rendement dans l'année d'adoption. Pour comprendre ce phénomène, deux explications ont été avancées précédemment. La première suggère que l'adoption résulte d'un choix légitime d'affaires tel qu'un besoin de financement pour nourrir cette croissance. La deuxième considère, plutôt, un choix opportuniste de la part des entreprises et suggère que l'adoption des IAS/IFRS peut servir d'opportunité afin de maquiller ou embellir la situation financière (exemple: refléter une meilleure croissance). Dans la première explication, la croissance est réelle, alors que dans la deuxième, elle est " artificielle ».

Il est possible de distinguer les deux explications en examinant l'historique de croissance des entreprises. Si la croissance dans l'année d'adoption suit le rythme historique de croissance, les entreprises

Comptabilité - Contrôle - Audit / Tome ??? - Volume ??? - ??? 2007 (p. ??? à ???) 
Houda AfFes et Antonello CALLimaCi

adoptant les normes de façon anticipée peuvent être plus difficilement accusées de maquiller leur situation financière et l'explication d'une raison légitime d'affaires, tel que le financement, gagne en crédibilité. Pour vérifier laquelle des deux explications est la plus plausible, une nouvelle variable de croissance excessive est créée, c'est-à-dire, la croissance supplémentaire au-delà de la croissance historique moyenne:

Croissance Excessive $=$ Croissance Réelle - Croissance Historique Moyenne

Si le but d'adopter les normes internationales était de majorer la croissance, la variable croissance excessive devrait être positivement associée à l'adoption anticipée dans notre modèle logistique. Afin de calculer la croissance excessive, il faut trouver la croissance historique. Pour ce faire, les croissances moyennes de l'actif total (TACT) et du rendement (ROE) sont prises pour chaque entreprise sur une période de trois années précédant l'année d'adoption ${ }^{15}$. La différence entre la croissance réelle au temps $t$ et celle historique moyenne (de $t-1$ à $t$-3) est intégrée dans le modèle logistique pour le total de l'actif et le rendement (respectivement les variables CTACT_Ex et CROE_Ex). Puisque des données pour le calcul de la moyenne historique sont manquantes pour 12 entreprises, l'échantillon est restreint à 94 observations pour ce test.

Les résultats du modèle intégrant la croissance excessive, présentés au tableau 2, confirment ceux du modèle de base. Les coefficients de la taille (CTACT), l'intensité capitalistique (ICAP), la croissance de la taille (CTACT) et celle du rendement (CROE) sont tous positifs et significatifs; seul le coefficient de la dispersion de l'actionnariat (DISP) n'est plus significatif.

Tableau 2

Analyse logistique des déterminants de l'adoption anticipée des normes IAS/IFRS Intégration de la croissance excessive

\begin{tabular}{|c|c|c|c|}
\hline \multicolumn{4}{|c|}{ 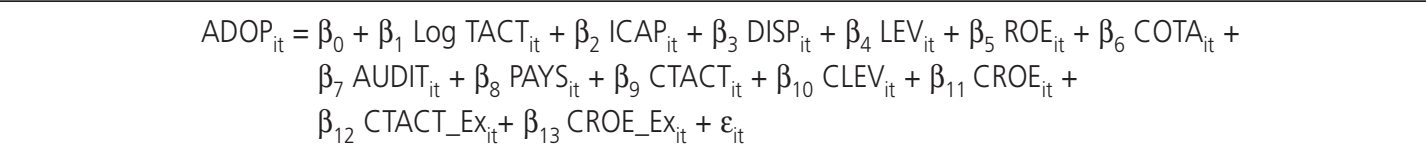 } \\
\hline Variable & Signe prévu & Coefficient $\beta$ & $p$ \\
\hline Log TACT & + & 1,393 & 0,009 \\
\hline ICAP & + & 0,054 & 0,008 \\
\hline DISP & + & 0,018 & 0,211 \\
\hline LEV & + & $-0,006$ & 0,829 \\
\hline ROE & + & $-0,021$ & 0,109 \\
\hline COTA & + & 0,220 & 0,838 \\
\hline AUDIT & + & $-0,335$ & 0,605 \\
\hline PAYS & $+/-$ & $-0,332$ & 0,634 \\
\hline CTACT & + & 0,030 & 0,018 \\
\hline CLEV & + & $-0,001$ & 0,594 \\
\hline CROE & + & 0,172 & 0,039 \\
\hline CTACT_EX & $+/-$ & $-0,003$ & 0,723 \\
\hline CROE_EX & $+/-$ & $-0,104$ & 0,143 \\
\hline \multicolumn{4}{|c|}{ Taux de prédiction non biaisé: $76,6 \% \quad P$ du modèle: 0,001} \\
\hline
\end{tabular}

Comptabilité - Contrôle - Audit / Tome ??? - Volume ??? - ??? 2007 (p. ??? à ???) 
Houda AfFes et Antonello CALLIMACI

LES DÉTERMINANTS DE L'ADOPTION ANTICIPÉE DES NORMES COMPTABLES INTERNATIONALES : CHOIX FINANCIER OU OPPORTUNISME?

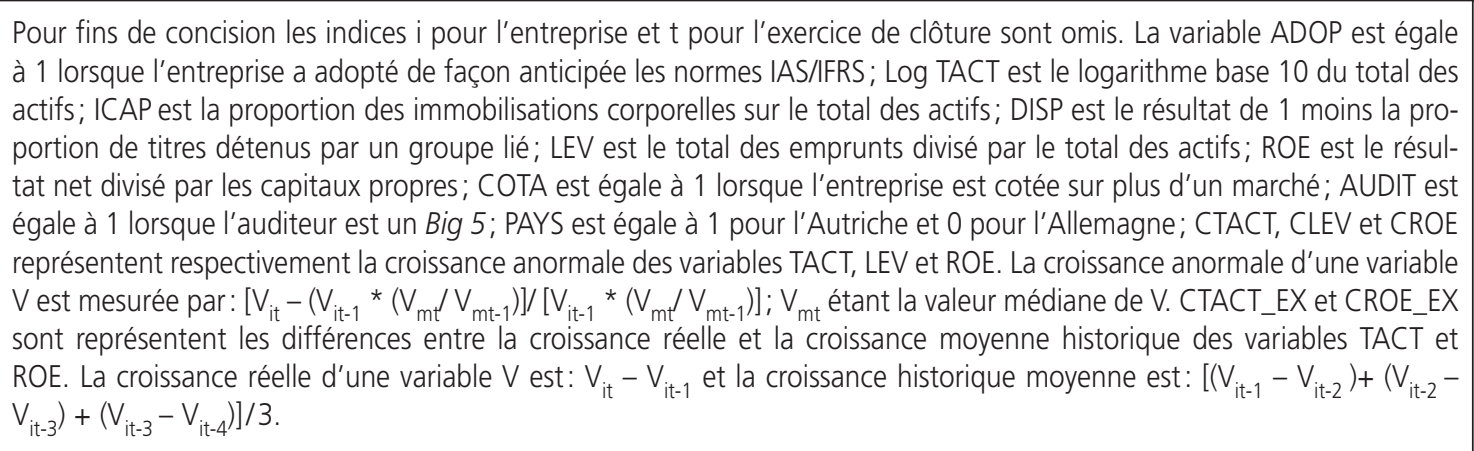

Les croissances excessives ne sont pas positivement associées à l'adoption anticipée des normes IAS/IFRS tel qu'exigé par l'hypothèse d'une adoption opportuniste dans le but de maquiller la situation financière. Non seulement ces croissances excessives ne sont pas significatives ( $p=0,723$ et 0,143 respectivement) pour l'actif total et le rendement (CTACT_Ex et CROE_Ex), mais leur coefficient est négatif (respectivement ${ }_{-12}=-0,003$ et ${ }_{-13}=-0,104$ ). Ceci renforce la conclusion que les normes IAS/IFRS ne sont pas adoptées dans le but d'embellir la situation financière en présentant des croissances accrues. Les résultats permettent de conclure que les firmes ayant adopté les normes internationales ont une croissance réelle et que l'adoption anticipée semble plutôt être justifiée par la recherche de sources supplémentaires de financement. Les motivations de l'adoption anticipée des normes IAS/IFRS concordent donc avec les objectifs visés par le normalisateur comptable international et ceux du parlement européen qui en a rendu l'utilisation obligatoire.

\section{Conclusion}

Alors que le règlement 1606/2002/ CE oblige les entreprises cotées sur les marchés de capitaux de l'UE à préparer leurs états financiers consolidés conformément aux normes IAS/IFRS pour les exercices débutant le premier janvier 2005 ou après, plusieurs entreprises domiciliées dans les pays membres les avaient déjà adoptées de façon anticipée. L’objectif de cette étude est d'identifier les principaux facteurs associés à cette adoption anticipée des normes IAS/IFRS.

Le modèle développé est en mesure de classer correctement environ $75 \%$ des observations. L'analyse révèle que les entreprises les plus favorables à l'adoption anticipée des IAS/IFRS en Allemagne et en Autriche sont de grande taille, ont une forte intensité capitalistique et ont un capital dispersé. De plus, elles présentent une plus forte croissance anormale de la taille et de la rentabilité que les entreprises choisissant de garder les normes locales. Ces résultats pris collectivement incitent à croire que la motivation de l'adoption anticipée est liée au financement. À priori, l'endogénéité de la relation entre la croissance et l'adoption anticipée des normes internationales ne peut être exclue; il est possible que la croissance de la taille et de la rentabilité ne soit pas la cause de l'adoption des normes internationales, mais plutôt la conséquence escomptée par une motivation de maquiller les états financiers. Des analyses additionnelles sont effectuées pour vérifier si l'adoption anticipée est due à une motivation de financement ou de manipulation des états financiers. Les résultats démontrent que l'adoption anticipée des IAS/IFRS n'est pas associée à un taux de croissance dans l'année d'adop- 
Houda AfFes et Antonello Callimaci

16 LES DÉTERMINANTS DE L'ADOPTION ANTICIPÉE DES NORMES COMPTABLES INTERNATIONALES: CHOIX FINANCIER OU OPPORTUNISME?

tion supérieur au taux historique des trois années précédentes. Il semble que les dirigeants décident d'adopter les normes internationales afin d'avoir accès à des ressources financières susceptibles d'alimenter la croissance de leur entreprise plutôt que de créer une croissance fictive.

Il est juste de noter que l'étude de deux pays est déjà une limite en soi à la validité externe. De plus, le pouvoir explicatif du modèle reste modeste. Ceci connote l'existence de variables omises dans le modèle qui pourraient être étudiées dans des recherches ultérieures. Les recherches futures pourraient, également, examiner l'impact économique de l'adoption des normes IAS/IFRS et l'inclure à titre de déterminant en l'intégrant dans le modèle logistique. 
Houda Affes et Antonello CALlimaCi LES DÉTERMINANTS DE L'ADOPTION ANTICIPÉE DES NORMES COMPTABLES INTERNATIONALES: CHOIX FINANCIER OU OPPORTUNISME?

\section{Bibliographie}

Al-BASTEKI H. (1995), "The voluntary adoption of international accounting standards by bahraini corporations ", Advances in International Accounting, volume 8, pp. 47-64.

AYRES F. (1986), "Characteristics of firms electing early adoption of SFAS 52 ", Journal of Accounting and Economics, volume 8, pp. 143-158.

BALl R., BROWN V. (1968), "An Empirical Evaluation of Accounting Income Numbers», Journal of Accounting Research, pp. 159-178.

Bartov E., Goldberg S.R., Kim M.S. (2002), "Comparative value Relevance among German, U.S. and international accounting standards: a German stock market perspective ", Document de recherche, janvier 2001, 26 pages.

BuzBy S. (1975), "Company size, listed versus unlisted stocks, and the extent of financial disclosure ", Journal of Accounting Research, volume 13, $\mathrm{n}^{\mathrm{o}} 1$, pp. 16-37.

Chow C., Wong-Boren A. (1987), «Voluntary financial disclosure by Mexican corporations ", The Accounting Review, volume 62, pp. 533-541.

Clarkson P.M., KaO J.L., Richardson G.D. (1994), "The voluntary inclusion of forecasts in MD\&A section of annual reports ", Contemporary Accounting research, volume 11, $\mathrm{n}^{\circ} 1$, pp. 423-451.

Colasse B. (2000), " Harmonisation comptable internationale ", Encyclopédie de comptabilité, contrôle de gestion et audit, pp. 757-769.

Cuijpers R., Buijink W., Majoor S. (2002), "Voluntary adoption of non-local GAAP in the European Union: a study of determinants ", Document de recherche, mars 2002, 31 pages.

Dumontier P., RAFFOURnier V. (1998), "Why firms comply voluntarily with IAS: an empirical analysis with Swiss data ", Journal of International Financial Management and Accounting, volume 9, $\mathrm{n}^{\mathrm{o}} 3$, pp. 216-245.

El-Gazzar S. M., Finn P. M., Jacob R. (1999), « An empirical investigation of multinational firms' compliance with international accounting standards ", The International Journal of Accounting, volume $34, \mathrm{n}^{\circ} 2$, pp. 239-248.
Hossain M., Tan L., Adams M. (1994), «Voluntary disclosure in an emerging capital market: some empirical evidence from companies listed on the Kuala Lumpur stock exchange ", The International Journal of Accounting, volume 29, pp. 334-351.

LANG M.H., LundHOLM R.J. (1993), "Cross sectional determinants of analyst ratings of corporate disclosures ", Journal of Accounting Research, volume 31, no 2, pp. 246-271.

LEUZ C., VERRECCHIA R.E. (2000), «The economic consequences of increased disclosure", Accounting Information and Firm Economics, volume 38, supplement, pp. 91-124.

Meek G., Roberts C., Gray S. (1995), " Factors influencing voluntary annual report disclosures by U.S., U.K. and continental European multinational corporations ", Journal of international business studies, volume 26, $\mathrm{n}^{\circ} 3$, pp. 555-572.

Mora A., ReEs W. (1998), "The early adoption of consolidated accounting in Spain ", The European Accounting Review, volume 7, no 4, pp. 675-696.

Murphy A.B. (1999), "Firm characteristics of Swiss companies that utilize international accounting standards ", The international Journal of Accounting, volume 34, $\mathrm{n}^{\circ}$ 1, pp. 121-131.

RAFFOURNiER B. (1995), "The determinants of voluntary financial disclosure by Swiss listed companies ", The European accounting review, volume 4, no 2, pp. 261-280.

SAmi H., Welsh M.J. (1992), "Characteristics of early and late adopters of pension accounting standard SFAS No. 87 ", Contemporary Accounting Research, volume 9, $\mathrm{n}^{\circ}$ 1, pp. 212-236.

ScotT T.W. (1991), " Pension disclosures under SFAS No. 87: theory and evidence ", Contemporary Accounting Research, volume 8, $\mathrm{n}^{\mathrm{o}} 1$, pp. 62-81.

Singhvi S., Desai H. (1971), «An empirical analysis of the quality of corporate financial disclosure ", The Accounting Review, volume 46, pp.129-138.

Street D.L., Gray S.J. (2002), « Factors influencing the extent of corporate compliance with international accounting standards: summary of a research monograph ", Journal of International Accounting Auditing and Taxation, volume 11, pp. 51-76. 
Houda Affes et Antonello CallimaCi LES DÉTERMINANTS DE L'ADOPTION ANTICIPÉE DES NORMES COMPTABLES INTERNATIONALES: CHOIX FINANCIER OU OPPORTUNISME?

Trombley M.A. (1989), "Accounting method choice in the software industry: characteristics of firms electing early adoption of SFAS No. 86 ", The Accounting Review, volume 64, no 3, pp. 529538.

Wallace R.S.O., Naser K. (1995), "Firm specific determinants of the comprehensiveness of mandatory disclosure in the annual corporate reports of firms listed on the stock exchange of Hong Kong ", Journal of Accounting and Public Policy, volume 14, pp. 311-368.

\section{Notes}

1. Les adoptions anticipées et volontaires ne représentent pas le même événement. Une adoption anticipée est une adoption qui a lieu avant qu'elle ne devienne obligatoire. Une adoption volontaire est entièrement facultative car elle n'est pas exigée par une norme.

2. Au moment de la collectes des données, les «Big 5 " étaient constitués par: Price Waterhouse Coopers, KPMG, Deloitte \& Touche, Arthur Andersen et Ernst \& Young.

3. À titre d'exemple, selon Bartov et al. (2002), les investisseurs des marchés boursiers allemands sont mieux informés lorsque l'information comptable répond aux exigences de divulgation des normes internationales qu'à celles des normes locales.

4. L'utilisation de deux autres mesures de la taille, le $\log$ du total des ventes nettes et le log de la valeur au marché de l'entreprise, n'affecte pas les résultats. Ces résultats ne seront pas discutés ni présentés par soucis de concision.

5. Un problème se pose quand la variable $V_{\text {it- } 1}$ est négative; évidemment cela est possible seulement pour le rendement. Dans ces rares cas, nous avons refait le calcul manuellement afin de refléter correctement la croissance anormale du rendement.

6. Aucune exception ni dérogation par rapport à une norme n'est indiquée.
7. L'entreprise utilise de façon ininterrompue les normes IAS/IFRS après leur adoption initiale.

8. Nous remercions un réviseur d'avoir attiré notre attention sur le fait que l'identification des normes comptables utilisées par les entreprises comporte parfois des inexactitudes dans Worldscope et qu'une vérification avec les rapports annuels s'avère nécessaire.

9. Pour les variables mesurant la taille, une entreprise n'est éliminée que si elle considérée aberrante pour au moins deux de ses trois mesures: le log du total de l'actif, le log du total des ventes nettes et le log de la valeur au marché de l'entreprise. Le poids d'une variable pour une entreprise i est mesuré par le ratio: $\mathrm{V}_{\text {it }} / \sum\left|\mathrm{V}_{\text {it }}\right|$; la somme des valeurs absolues étant calculée pour l'échantillon au complet.

10. Le fait que l'échantillon final soit équilibré est un hasard. En éliminant les observations aberrantes, neuf (9) observations ont été exclues de chaque groupe. Afin de ne pas trop restreindre l'échantillon, les paires d'observations n'ont pas été exclues, c'est-à-dire du groupe contrôle et expérimental. Des tests de robustesse utilisant un échantillon équilibré et parfaitement apparié produisent des résultats similaires.

11. Le tableau n'est pas présenté par souci de concision.

12. Les résultats demeurent inchangés pour toutes les variables, sauf celle AUDIT qui devient significative. Les résultats sont discutés en détail dans les sections suivantes.

13. Le tableau n'est pas présenté par souci de concision.

14. Comme par exemple, Grant Thornton et BDO.

15. Nous excluons de ce test additionnel la variable de la croissance de l'endettement (CLEV) car le test de base révèle que cette variable est non significative. 